\title{
Ultrafast Adsorbate Excitation Probed with Subpicosecond-Resolution X-Ray Absorption Spectroscopy
}

\author{
Elias Diesen $\odot,{ }^{1, *}$ Hsin-Yi Wang, ${ }^{2}$ Simon Schreck, ${ }^{2}$ Matthew Weston, ${ }^{2}$ Hirohito Ogasawara, ${ }^{3}$ Jerry LaRue, ${ }^{4}$ \\ Fivos Perakis, ${ }^{2}$ Martina Dell'Angela, ${ }^{5}$ Flavio Capotondi, ${ }^{6}$ Luca Giannessi, ${ }^{6}$ Emanuele Pedersoli, ${ }^{6}$ \\ Denys Naumenko, ${ }^{6}$ Ivaylo Nikolov, ${ }^{6}$ Lorenzo Raimondi, ${ }^{6}$ Carlo Spezzani ${ }^{6}$ Martin Beye, ${ }^{7}$ Filippo Cavalca, ${ }^{2}$ \\ Boyang Liu, ${ }^{2}$ Jörgen Gladh, ${ }^{2,3}$ Sergey Koroidov, ${ }^{2}$ Piter S. Miedema, ${ }^{7}$ Roberto Costantini, ${ }^{5,8}$ Tony F. Heinz, ${ }^{3,9}$ \\ Frank Abild-Pedersen, ${ }^{1}$ Johannes Voss, ${ }^{1}$ Alan C. Luntz, ${ }^{1}$ and Anders Nilsson ${ }^{2}$ \\ ${ }^{1}$ SUNCAT Center for Interface Science and Catalysis, SLAC National Accelerator Laboratory, \\ 2575 Sand Hill Road, Menlo Park, California 94025, USA \\ ${ }^{2}$ Department of Physics, AlbaNova University Center, Stockholm University, SE-10691 Stockholm, Sweden \\ ${ }^{3}$ SLAC National Accelerator Laboratory, 2575 Sand Hill Road, Menlo Park, California 94025, USA \\ ${ }^{4}$ Schmid College of Science and Technology, Chapman University, Orange, California 92866, USA \\ ${ }^{5}$ CNR-IOM, SS 14-km 163.5, 34149 Basovizza, Trieste, Italy \\ ${ }^{6}$ FERMI, Elettra-Sincrotrone Trieste, SS 14-km 163.5, 34149 Basovizza, Trieste, Italy \\ ${ }^{7}$ DESY Photon Science, Notkestrasse 85, Hamburg 22607, Germany \\ ${ }^{8}$ Physics Department, University of Trieste, Via Valerio 2, 34127 Trieste, Italy \\ ${ }^{9}$ Department of Applied Physics, Stanford University, Stanford, California 94305, USA
}

(Received 22 December 2020; accepted 7 May 2021; published 29 June 2021)

\begin{abstract}
We use a pump-probe scheme to measure the time evolution of the $\mathrm{C} K$-edge $\mathrm{x}$-ray absorption spectrum from $\mathrm{CO} / \mathrm{Ru}(0001)$ after excitation by an ultrashort high-intensity optical laser pulse. Because of the short duration of the x-ray probe pulse and precise control of the pulse delay, the excitation-induced dynamics during the first picosecond after the pump can be resolved with unprecedented time resolution. By comparing with density functional theory spectrum calculations, we find high excitation of the internal stretch and frustrated rotation modes occurring within $200 \mathrm{fs}$ of laser excitation, as well as thermalization of the system in the picosecond regime. The $\sim 100 \mathrm{fs}$ initial excitation of these $\mathrm{CO}$ vibrational modes is not readily rationalized by traditional theories of nonadiabatic coupling of adsorbates to metal surfaces, e.g., electronic frictions based on first order electron-phonon coupling or transient population of adsorbate resonances. We suggest that coupling of the adsorbate to nonthermalized electron-hole pairs is responsible for the ultrafast initial excitation of the modes.
\end{abstract}

DOI: 10.1103/PhysRevLett.127.016802

The vibrational, rotational, and translational motions of adsorbates are the fundamental physical processes behind chemical reactions on surfaces. Understanding the details of adsorbate dynamics can therefore be essential for describing heterogeneous catalytic reactions, with the eventual goal of tailoring catalysts for high efficiency and selectivity. In particular, how energy is transferred from the bulk substrate to the adsorbate modes is key to understanding selectivity in catalysis. Since surface chemical reactions proceed through several elementary dynamical steps on different timescales in parallel with competing energy relaxation processes, studying these in a timeresolved manner can give knowledge about the elementary adsorbate-surface interaction and energy transfer processes.

Exciting an adsorbate-substrate system with an intense femtosecond optical pulse and then probing the dynamical evolution of the adsorbate by time-resolved optical techniques, such as sum-frequency generation (SFG) [1-6] and two-pulse correlation (2PC) [7-9] has a modest history.
More recently, x-ray absorption and emission spectroscopy (XAS and XES) [10-14], has also become a powerful approach for gaining insight into the transient excitation of adsorbate vibrations and the consequent diffusion, desorption, and final chemical reactions. X-ray spectroscopies are inherently element-specific local probes and thus particularly suited for studying any process that affects the electronic structure and chemical environment of a specific atom [15]. In particular, an X-ray free-electron laser (XFEL) delivering femtosecond $\mathrm{x}$-ray pulses allows probing dynamical changes of the adsorbate electronic structure induced by the femtosecond optical laser. Activation of atomic oxygen [12] and carbon monoxide [10], as well as driving of CO oxidation [13], are examples of reactions that have been studied with this technique.

In this work, we report experimental $\mathrm{C} K$-edge XAS of $\mathrm{CO} / \mathrm{Ru}(0001)$ during and after pulsed optical laser excitation, probed with a time resolution of $\sim 100 \mathrm{fs}$ in a conventional pump-probe setup. This is possible due to the 
ultrashort duration of XFEL pulses (at DIPROI beamline of the FERMI facility in Elettra, Italy). The XFEL is seeded by a tunable solid state laser system and the photon energy is multiplied through a two-stage high-gain harmonic generation process [16]. The polarized soft x-ray pulses with less than $50 \mathrm{fs}$ duration are delivered to the sample at a repetition rate of $10 \mathrm{~Hz}$ as the "probe." Meanwhile, a portion of the seed laser is separated and coupled into the experimental chamber collinearly with the $\mathrm{x}$ rays, acting as the "pump" with the same repetition rate. Since the seed and the pump originate from the same laser pulse, the time jitter between the pump laser and the XFEL $\mathrm{x}$-ray pulse is less than $6 \mathrm{fs}$ [17]. The aforementioned properties allow direct detection of the adsorbate electron structure as influenced by internal excitations before the substrate phonons have been excited, which takes around 1 ps. Since $\mathrm{CO}$ chemisorbs on $\mathrm{Ru}(0001)$ in an upright geometry at the on-top site (with the $\mathrm{C}$ atom coordinated toward the $\mathrm{Ru}$ atom) [18], the dipole selection rule gives the strongest moment for the $\mathrm{C} K$-edge $1 s \rightarrow 2 \pi^{*}$ transition when the electric field vector ( $E$ vector) is parallel to the surface. Using linearly polarized $\mathrm{x}$ rays and measuring with the $E$ vector oriented either in plane (lin $\mathrm{H}$ ) or out of plane (lin V) with respect to the metal surface, we can thus monitor how the electronic structure and molecular orientation temporally change following the optical laser pumping. The optical laser is p-polarized, precluding any direct optical excitation of the $2 \pi^{*}$. The C $K$ edge, due to similar intramolecular potential energy curves in the ground and core-excited states, gives only small Franck-Condon factors for $\mathrm{C}-\mathrm{O}$ vibrational excitation in the $\mathrm{x}$-ray transition $[19,20]$, and thereby provides excellent spectral contrast in comparison to the much broader $\mathrm{O} K$ edge used in most previous work [10,11,21].

Recently, $\mathrm{CO}$ desorption from $\mathrm{CO} / \mathrm{Ru}$ was studied on picosecond timescales, and a weakly adsorbed precursor state $[11,22]$ was directly identified and its evolution described [14]. In this Letter, we focus on the ultrafast dynamics occurring in the $\mathrm{CO}$ within the very first picosecond following optical laser excitation of the $\mathrm{Ru}$. The high spectral resolution and controlled pump-probe delay, together with detailed spectrum simulations, allow us to identify the excitation of specific adsorbate modes and their initial excitation times. We find significantly faster energy transfer to the adsorbate than conventional nonadiabatic theoretical approaches suggest, and we postulate that nonthermal electron-hole $(e-h)$ pairs initially created by the optical laser may account for the ultrafast initial excitations of these modes.

The $\mathrm{Ru}(0001)$ crystal was first cleaned by standard procedures [14], and a saturated CO adlayer ( 0.66 ML) on Ru was prepared by pressurizing the UHV chamber with $1.0 \times 10^{-8}$ torr of $\mathrm{CO}$ while cooling the crystal down to liquid nitrogen temperature [23]. The crystal sample was pumped using $400 \mathrm{~nm}$ optical laser pulses (fluence of $\sim 91 \mathrm{~J} / \mathrm{m}^{2}$ ) at a grazing incidence angle of around $2^{\circ}$ with duration of $100 \mathrm{fs}$. A fresh sample spot for each shot was realized by continuously scanning the sample. A CO background pressure of $1.0 \times 10^{-8}$ torr was used to ensure a fully recovered surface adlayer once the same spot was revisited after scanning the complete sample. XAS was recorded by detecting $\mathrm{x}$-ray fluorescence, using a previously described detector setup [24], and the incident energy was stepwise changed across the carbon $1 s \rightarrow 2 \pi^{*}$ resonance edge in the range of 285-290 eV. The XAS was thus plotted by averaging the total number of emitted photons recorded as a function of the incident energy for every desired pump-probe delay. The definition of time zero delay is discussed in Supplemental Material, A [25]. Further details of the experimental method can be found elsewhere [14].

The top panel of Fig. 1 shows the lin H XAS spectra evolving with pump-probe delay time. The optical unpumped spectrum (averaged between -300-0 fs) depicts a strong C $1 s \rightarrow 2 \pi^{*}$ resonance peak centered at around $288 \mathrm{eV}$. For delays $0-100$ fs following the laser pump pulse, a sudden decrease of intensity at the peak center and an increase of intensity on the lower energy side of the spectrum is clearly seen, while the main peak energy stays the same. However, in the next 100 fs delay a redshift

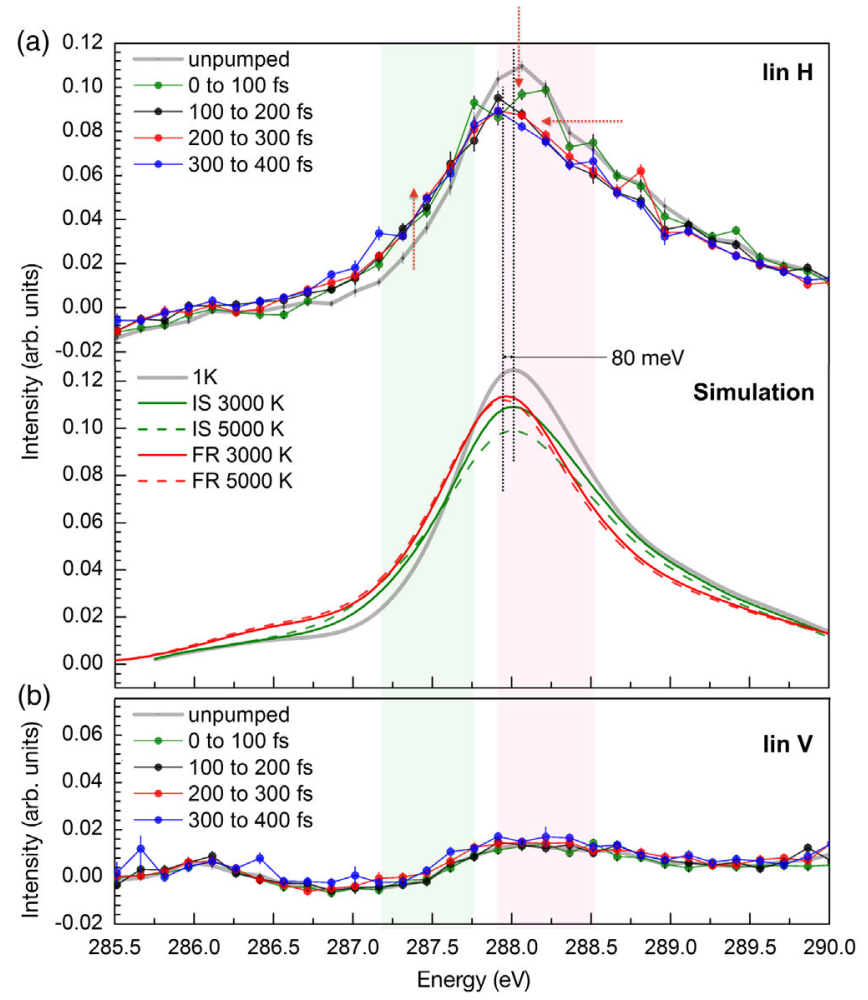

FIG. 1. (a) Measured lin H spectra, averaged over the indicated pump-probe delay ranges, together with calculated spectra with excited internal stretch (IS) and frustrated rotation (FR) modes. (b) Measured lin V spectra. (The negative intensity is due to background subtraction.) 
of about $80 \mathrm{meV}$ of the peak center can be identified. No further rapid changes are found at later times, indicating a slower energy equilibration after the initial excitation. The lin V spectra [Fig. 1(b)], on the other hand, indicates that the aforementioned observation in the lin $\mathrm{H}$ spectrum at 0-100 fs delay cannot be ascribed to the molecule tilting since no corresponding intensity increasing at $288 \mathrm{eV}$ can be seen in the lin V spectrum. Instead, a mild increase of overall intensity in lin $\mathrm{V}$ spectra can be seen in the following delays, up to about 1 ps.

To study the time evolution in detail, the intensities are integrated over two energy regions (main peak region in the pink area and lower energy region in the green area) chosen in order to give a clear view of the time dependence. The time evolution of these integrated intensities is shown in Fig. 2. In addition, the lin $\mathrm{H}$ and lin $\mathrm{V}$ spectra at longer time delays are shown in Supplemental Material, B [25]. We first focus on the main peak region. Figure 2(a) shows a sudden decrease of intensity in the first 0-100 fs and 100-200 fs delays of the lin $\mathrm{H}$ spectrum due to the main peak intensity dropping and peak shifting, respectively [cf. Fig. 1(a)], followed by a gradually decreasing intensity after 200 fs and a corresponding increasing intensity in the lin $\mathrm{V}$ spectrum with time. On the lower energy side [Fig. 2(b)], a sudden increase of intensity in the first $0-100$ fs delay followed by a gentle increase of intensity after $100 \mathrm{fs}$ can be observed in the lin $\mathrm{H}$ spectra, while for lin V spectra no obvious changes in 0-100 fs delay can be seen, yet a similar intensity increasing trend as in the lin $\mathrm{H}$ spectra shows up after 100 fs. We can thus distinguish two processes occurring on different timescales, one very rapid which is completed after around 200 fs and mainly affects the lin $\mathrm{H}$ spectrum, and one slower affecting both polarizations.
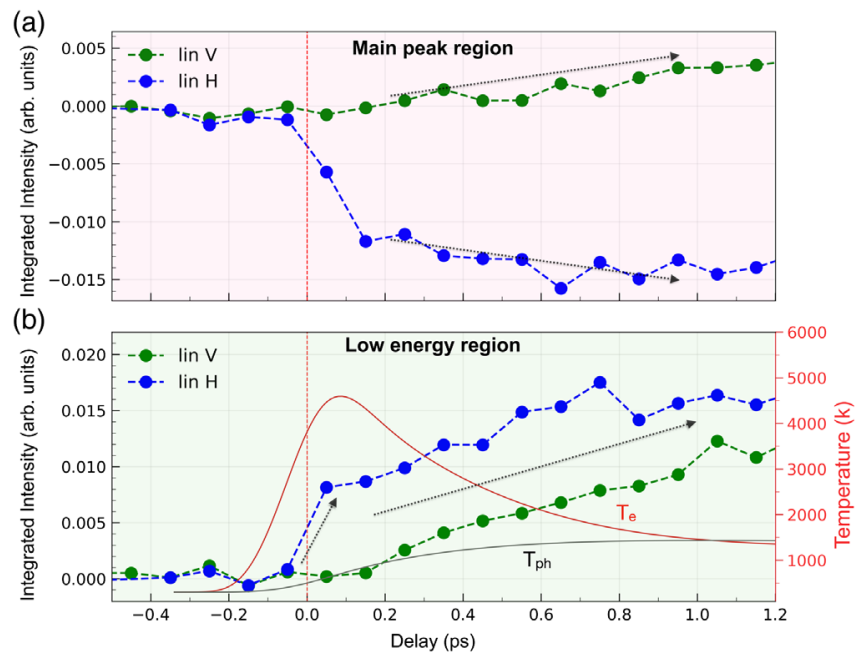

FIG. 2. Time evolution of the integrated spectral intensity, minus the unpumped intensity, in (a) the main peak region and (b) low energy region, respectively (cf. Fig. 1). Also shown in (b) are the electron $\left(T_{e}\right)$ and phonon $\left(T_{\mathrm{ph}}\right)$ temperatures from the two-temperature model.
To interpret the time evolution of the spectra, we perform density functional theory (DFT) calculations using the Quantum ESPRESSO [42,43] code, with spectra calculated using the xspectra [44-46] code. The general method for spectral calculation and treatment of the core-hole has been described elsewhere [47]. A $4 \times 4 \times 4$ periodic slab was used for all spectrum calculations with a single $\mathrm{CO}$ molecule adsorbed at an on-top site, corresponding to $1 / 4 \mathrm{ML}$ coverage instead of the experimental 0.66 ML. However, we have checked that our spectroscopy simulations are not sensitive to the absolute coverage (see Supplemental Material, F [25]). For further computational details, see Supplemental Material, C [25].

Previous theoretical studies of the first order electronphonon coupling of $\mathrm{CO}$ adsorbed on transition metal surfaces, e.g., $\mathrm{CO} / \mathrm{Cu}(100), \mathrm{CO} / \mathrm{Ni}(111)$ and $\mathrm{CO} / \mathrm{Pt}(111)$, suggested that the internal C-O stretch (IS) and the frustrated $\mathrm{CO}$ rotation (FR) modes are the modes most likely to be significantly excited directly by thermal metal electronhole pair excitations [48]. Our theoretical studies of the first order electron-phonon coupling for $\mathrm{CO} / \mathrm{Ru}$ (see Supplemental Material, K [25]) confirm this pattern, with the IS exhibiting the strongest coupling followed by that for FR. To calculate the XAS for internal stretch vibrationally excited states of the $\mathrm{CO}$ molecule at the surface, we determine the potential energy curve for the electronic ground state by varying the $\mathrm{C}-\mathrm{O}$ distance and calculating the DFT total energy for each point. We then fit a Morse potential to the data, which gives the eigenstates and an analytical representation of the vibrational eigenfunctions. The potential for the internal stretch mode with coreexcited $\mathrm{C}$ was determined in the same way, with the $\mathrm{C}$ pseudopotential containing an explicit core-hole. The unit cell was kept neutral by adding an electron at the Fermi level, since the metal electrons screen the core-hole very rapidly [49]. The Franck-Condon factors for the C $1 s \rightarrow$ $2 \pi^{*}$ transition can then be calculated analytically. We calculate the spectra at several displacements and average them together with the appropriate weight for the vibrational eigenstate to get the spectrum for each vibrational state (for details, see Supplemental Material, D and E [25]). The frustrated rotation mode was treated similarly, but with a harmonic potential assumed for the description of the mode. Finally, the spectra for the different vibrational states were added together, with weights given by the Boltzmann distribution for a desired temperature.

The calculated spectra for different vibrational modes at various thermal excitations are shown in Fig. 1. We first note that the agreement of the ground state theoretical spectrum with the experimental spectrum at negative delay is excellent (see also Fig. S4), giving confidence to the spectrum simulations. The calculated spectra for elevated temperatures of the internal stretch (IS) mode show a broadening of the peak and decrease of the maximum intensity, however the peak position remains unchanged. 
High temperature in the frustrated rotation (FR) mode on the other hand gives an $80 \mathrm{meV}$ redshift, in excellent quantitative agreement with the experimental results for $\sim 200$ fs delay. We therefore identify the IS excitation as inducing the initial decrease of the peak and the FR excitation as causing the redshift of the peak. It might be expected that FR motion would also give a strong signal in the vertical polarization direction, since tilting the molecule means the $\mathrm{C} 1 s \rightarrow 2 \pi^{*}$ dipole moment will have nonzero projection in the vertical direction. However, as seen also in Fig. S5 [25] the actual response is quite small. During pure FR excitation the molecular center-of-mass remains fixed, and there is no simple direct relation between the two polarization signals (Fig. S5). In addition, the slow increase we see in the vertical signal over the first picosecond is consistent with the anticipated complicated dynamics en route to overall thermalization of the system. For comparison, the results of the commonly used twotemperature model are also shown in Fig. 2(b) (see also Supplemental Material, G [25]). It is immediately clear that the initial response in the lin $\mathrm{H}$ signal (blue dotted line) is faster than the rise of the phonon temperature (black line), and thus must be electron-driven, while the lin $\mathrm{V}$ signal (green dots) increase is due to more complicated dynamics involving both electrons and phonons (see discussion later). After $\sim 1$ ps, many phonons are excited and the surface geometry becomes much less well defined [3]. There is therefore a large number of adsorption sites and rotational states available, giving a slow increase of resonance intensity projected on the vertical direction (lin V) and a complementarily decreasing intensity in lin $\mathrm{H}$ spectra in the main peak region at $0.2-1$ ps delay [Fig. 2(a)]. The spectral changes at 1 ps can be qualitatively reproduced by simulating spectra from $a b$ initio molecular dynamics at a temperature of $1300 \mathrm{~K}$ (see Supplemental Material, $\mathrm{H}$ [25]), suggesting that near equilibration between all modes occurs by $\sim 1 \mathrm{ps}$. Desorption starts to be visible in the spectrum after a few picoseconds [14], beyond the time range studied in this Letter.

The mechanism behind the very fast adsorbate initial IS and FR excitation, which occurs within $200 \mathrm{fs}$, is unclear. First order electron-phonon coupling calculations of the frictional damping of thermalized metal electron-hole pairs suggest vibrational lifetimes $\tau \sim 2-4$ ps for the IS and FR modes for CO/Ru (see Supplemental Material, K [25]). A simple one dimensional classical master equation approach indicates that a coupling strength of $\geq 30 \mathrm{~cm}^{-1}$ (corresponding to $\tau \sim 200 \mathrm{fs}$ ) would be needed to obtain an energy transfer fast enough to describe the observed initial excitations in these modes (Supplemental Material, I [25]). We suggest that the most likely reason for the rapid initial excitation is that the highly excited nonthermal $e-h$ pair distribution, initially created in the substrate by laser absorption, could give a stronger coupling to adsorbate motion than friction models based on first order electron-phonon coupling where a thermalized $e-h$ pair bath is assumed to excite the adsorbate. Possible evidence for the contribution of nonthermalized $e-h$ pair to adsorbate excitation has previously been suggested for oxygen activation in a $\mathrm{O} / \mathrm{CO} / \mathrm{Ru}$ system [50], where the nonthermalized electron population right after optical excitation coupled more efficiently to an unoccupied adsorbate resonance (LUMO) compared to a thermalized hot FermiDirac distribution, which does not necessarily form within the first 100 fs [51].

The LUMO $2 \pi^{*}$ resonance for $\mathrm{CO} / \mathrm{Ru}$ is found around $5 \mathrm{eV}$ above the Fermi level [52], while the optical laser energy is only $3.1 \mathrm{eV}$ (i.e., $400 \mathrm{~nm}$ ). Even though the $2 \pi^{*}$ resonance is broad and a remnant of its resonance could extend to $3.1 \mathrm{eV}$, significant direct population of this resonance-as in ion-resonance models responsible for desorption induced by (multiple) electronic transitions [53] (DIET and DIMET) - is highly unlikely to give efficient energy transfer. Even if we assumed that the adsorbate LUMO $2 \pi^{*}$ resonance could be populated (e.g., by photoexcitation of initially created hot electrons in the laser pulse) we find that a simple dynamical model gives only little energy transfer into the motion of the adsorbate (Supplemental Material, J [25]), thus making resonant population of the adsorbate LUMO an inefficient excitation mechanism for $\mathrm{CO} / \mathrm{Ru}$ at $400 \mathrm{~nm}$. However, the nonthermal $e-h$ pair distribution could still excite adsorbate modes more efficiently than thermal $e-h$ pairs, since $e-h$ pair states far from the Fermi level may couple more strongly than close to it.

In this Letter, we only discuss the anomalous fast initial excitations of the IS and FR modes observed in the C XAS spectra compared to those predicted by first order electronphonon coupling, and the description after full thermalization. We make no attempt to describe the route between the two as that is driven by complicated coupled electron and phonon dynamics. Theoretical studies of the temperature dependence of the $\mathrm{CO} / \mathrm{Cu}(100)$ IS line shape and its evolution following femtosecond optical laser excitation even imply that first order electron-phonon coupling is not sufficient to describe the temporal dynamics of the coherently excited $v=1$ state of IS [54,55]. Extending the electron-phonon coupling to second order opens up an entirely new important class of energy transfer processes, so-called electron-mediated phonon-phonon couplings (EMPPC). While we have no definitive argument that these EMPPC processes are as important for $\mathrm{CO} / \mathrm{Ru}$ as for $\mathrm{CO} / \mathrm{Cu}(100)$, we believe that they likely are so that a complete understanding of the dynamics occurring between initial excitation and the fully thermalized system will ultimately require a multidimensional dynamical model including all of the EMPPC and direct phonon couplings. However, it is unlikely that their addition can account for the ultrafast initial mode excitations responsible for the changes in the XAS. For $\mathrm{CO} / \mathrm{Cu}(100)$, the fastest decay 


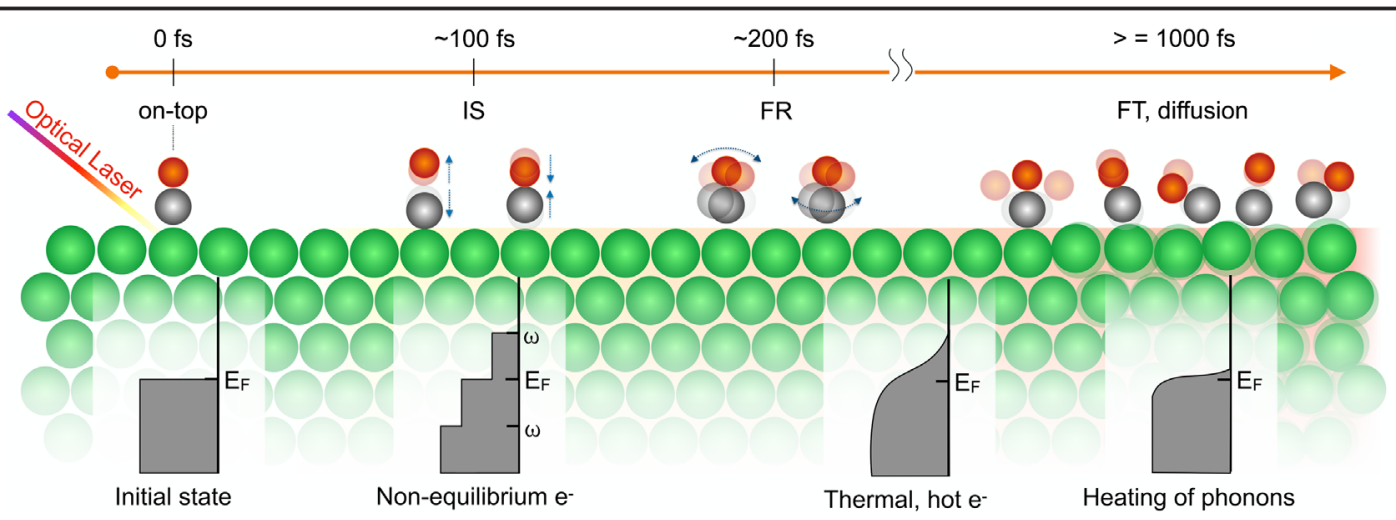

FIG. 3. Sketch of the system evolution during the first picosecond. Atom colors: O, red; C, gray; Ru, green.

processes for the wave vector $\boldsymbol{q} \sim 0, v=1$ state of IS are due to the first order electron-phonon coupling and dephasing of the $\boldsymbol{q} \sim 0$ into other $\boldsymbol{q}$ states of the same IS vibration (denoted as IS $\rightarrow$ IS) [55]. However, unlike photon excitation of an adsorbate vibrational mode, $e-h$ pair scattering excites the adsorbate modes with all $\boldsymbol{q}$. In addition, since XAS is a local probe at the C, the XAS spectrum is independent of the phase of vibrations contributing to the line shape so that the IS $\rightarrow$ IS process does not contribute to the dynamics observed. Therefore, if EMPPC in $\mathrm{CO} / \mathrm{Ru}$ are similar to those in $\mathrm{CO} / \mathrm{Cu}$, inclusion of EMPPC does not rationalize the ultrafast initial changes to the XAS compared to those predicted by first order electron-phonon coupling. Since optically created electron-hole pairs take 100 fs to thermalize in $\mathrm{Ru}$ [51], we instead suggest that nonthermalized electronhole pairs within the first $\sim 100$ fs have a stronger coupling to the adsorbate and preferentially excite the IS and FR modes within this timescale.

We summarize our main observations in Fig. 3. We can tentatively identify an initial excitation of the IS within $100 \mathrm{fs}$ of the laser pulse, followed by excitation of the FR mode during the subsequent $100 \mathrm{fs}$. Thermalization of the substrate phonons, and heating of the adsorbate translational modes, proceeds over the first ps, consistent with the commonly used two-temperature model. The initial excitations are significantly faster than what can be accounted for in nonadiabatic friction models with theoretically obtained friction coefficients, pointing to a lack of current theoretical descriptions of the coupling between highly excited electron distributions and adsorbate dynamics. Our observation is, however, in line with what has been inferred previously from experimental SFG [2,3,5,6] and 2PC [9] studies. A detailed explanation poses a challenge for future theoretical and experimental work.

This study shows the possibilities of the subpicosecond time resolution allowed by the well-controlled pump-probe delay in a seeded FEL setup. Compared to the 2PC technique, where subpicosecond dynamics can only be inferred indirectly by its influence on subsequent dynamics, and to SFG, where one can only probe a specific degree of freedom (typically the IS mode at $\boldsymbol{q} \sim 0$ ), this technique gives detailed information about the immediate response of the adsorbate local electronic structure to the driving pulse. We have thus been able to directly measure the evolution with time as it occurs, and together with DFT spectral calculations this allows a detailed analysis of the initial excitation and fully thermalized state. We expect that similar methods could be used to investigate the questions raised in this work regarding the role of nonthermal electrons and the seemingly strong frictional coupling.

This research was supported by the Knut and Alice Wallenberg Foundation under Grant No. 2016.0042, the Swedish Research Council under Grant No. 2013-8823, U.S. Department of Energy, Office of Science, Office of Basic Energy Sciences, Chemical Sciences, Geosciences, and Biosciences Division, Catalysis Science Program to the Ultrafast Catalysis FWP 100435 at SLAC National Accelerator Laboratory under Contract No. DE-AC0276SF00515. This research used resources of the National Energy Research Scientific Computing Center, a DOE Office of Science User Facility supported by the Office of Science of the U.S. Department of Energy under Contract No. DE-AC02-05CH11231. The authors acknowledge the continuous support of FERMI team during the setting and the operation of the FEL source for the experiment. M. B and P.S. M acknowledge funding from the Helmholtz association (VH-NG-1005). M.D. A and R.C. acknowledge support from the SIR Grant No. SUNDYN (Nr RBSI14G7TL, CUP B82I15000910001) of the Italian MIUR.

E. D. and H.-Y. W. contributed equally to this work.

*ediesen@stanford.edu

[1] M. Bonn, C. Hess, S. Funk, J. H. Miners, B. N. J. Persson, M. Wolf, and G. Ertl, Phys. Rev. Lett. 84, 4653 (2000).

[2] F. Fournier, W. Zheng, S. Carrez, H. Dubost, and B. Bourguignon, J. Chem. Phys. 121, 4839 (2004). 
[3] E. H. G. Backus, A. Eichler, A. W. Kleyn, and M. Bonn, Science 310, 1790 (2005).

[4] C. Hess, M. Bonn, S. Funk, and M. Wolf, Chem. Phys. Lett. 325, 139 (2000).

[5] K.-i. Inoue, K. Watanabe, and Y. Matsumoto, J. Chem. Phys. 137, 024704 (2012).

[6] K.-i. Inoue, K. Watanabe, T. Sugimoto, Y. Matsumoto, and T. Yasuike, Phys. Rev. Lett. 117, 186101 (2016).

[7] M. Bonn, D. N. Denzler, S. Funk, M. Wolf, S.-S. Wellershoff, and J. Hohlfeld, Phys. Rev. B 61, 1101 (2000).

[8] M. Bonn, S. Funk, C. Hess, D. N. Denzler, C. Stampfl, M. Scheffler, M. Wolf, and G. Ertl, Science 285, 1042 (1999).

[9] J. Gladh, T. Hansson, and H. Öström, Surf. Sci. 615, 65 (2013).

[10] M. Dell'Angela et al., Science 339, 1302 (2013).

[11] M. Beye et al., Phys. Rev. Lett. 110, 186101 (2013).

[12] M. Beye et al., J. Phys. Chem. Lett. 7, 3647 (2016).

[13] H. Öström et al., Science 347, 978 (2015).

[14] H. Wang et al., Phys. Chem. Chem. Phys. 22, 2677 (2020).

[15] A. Nilsson, J. Electron Spectrosc. Relat. Phenom. 126, 3 (2002).

[16] A. L. Yu et al., Science 289, 932 (2000).

[17] M. B. Danailov et al., Opt. Express 22, 12869 (2014).

[18] H. Over, W. Moritz, and G. Ertl, Phys. Rev. Lett. 70, 315 (1993).

[19] A. Föhlisch, J. Hasselström, O. Karis, D. Menzel, N. Mårtensson, and A. Nilsson, J. Electron Spectrosc. Relat. Phenom. 103-103, 303 (1999).

[20] O. Björneholm, A. Nilsson, E. O. F. Zdansky, A. Sandell, B. Hernnäs, H. Tillborg, J. N. Andersen, and N. Mårtensson, Phys. Rev. B 46, 10353 (1992).

[21] H. Xin et al., Phys. Rev. Lett. 114, 156101 (2015).

[22] H. Öberg et al., Surf. Sci. 640, 80 (2015).

[23] A. Föhlisch, W. Wurth, M. Stichler, C. Keller, and A. Nilsson, J. Chem. Phys. 121, 4848 (2004).

[24] J. Nordgren, G. Bray, S. Cramm, R. Nyholm, J.-E. Rubensson, and N. Wassdahl, Rev. Sci. Instrum. 60, 1690 (1989).

[25] See Supplemental Material at http://link.aps.org/supplemental/ 10.1103/PhysRevLett.127.016802 for experimental data; details of spectrum calculations; discussion of coverage dependence; details on the $2 T$ model; molecular dynamics results; models of energy transfer; and calculation of adsorbate vibration lifetimes, which includes Refs. [26-41].

[26] S. Mallikarjun Sharada, R. K. B. Karlsson, Y. Maimaiti, J. Voss, and T. Bligaard, Phys. Rev. B 100, 035439 (2019).

[27] D. Vanderbilt, Phys. Rev. B 41, 7892 (1990).

[28] L. Triguero, L. G. M. Pettersson, and H. Ågren, Phys. Rev. B 58, 8097 (1998).

[29] M. Leetmaa, M. P. Ljungberg, A. Lyubartsev, A. Nilsson, and L. G. M. Pettersson, J. Electron Spectrosc. Relat. Phenom. 177, 135 (2010).

[30] S. Flügge, Practical Quantum Mechanics (Springer-Verlag, Berlin Heidelberg, 1971).
[31] S. I. Anisimov, B. L. Kapeliovich, and T. L. Perel'man, Zh. Eksp. Teor. Fiz. 66, 776 (1974) [Sov. Phys. JETP 39, 375 (1974)].

[32] G. Kresse and J. Furthmüller, Phys. Rev. B 54, 11169 (1996).

[33] J. A. Misewich, T. F. Heinz, P. Weigand, and A. Kalamarides, Femtosecond Surface Science: The Dynamics of Desorption, in Laser Spectroscopy and Photochemistry on Metal Surfaces, edited by H.-L. Dai and W. Ho (World Scientific Publishing Company, Singapore, 1995), p. 764.

[34] J. Gavnholt, T. Olsen, M. Engelund, and J. Schiøtz, Phys. Rev. B 78, 075441 (2008).

[35] J. J. Mortensen, L. B. Hansen, and K. W. Jacobsen, Phys. Rev. B 71, 035109 (2005).

[36] J. Enkovaara et al., J. Phys. Condens. Matter 22, 253202 (2010).

[37] S. Baroni, S. de Gironcoli, A. Dal Corso, and P. Giannozzi, Rev. Mod. Phys. 73, 515 (2001).

[38] M. Calandra and F. Mauri, Phys. Rev. B 71, 064501 (2005).

[39] M. Wierzbowska, S. de Gironcoli, and P. Giannozzi, arXiv: cond-mat/0504077.

[40] A. C. Luntz, M. Persson, S. Wagner, C. Frischkorn, and M. Wolf, J. Chem. Phys. 124, 244702 (2006).

[41] D. Novko, M. Alducin, M. Blanco-Rey, and J. I. Juaristi, Phys. Rev. B 94, 224306 (2016).

[42] P. Giannozzi et al., J. Phys. Condens. Matter 21, 395502 (2009).

[43] P. Giannozzi et al., J. Phys. Condens. Matter 29, 465901 (2017).

[44] M. Taillefumier, D. Cabaret, A.-M. Flank, and F. Mauri, Phys. Rev. B 66, 195107 (2002).

[45] C. Gougoussis, M. Calandra, A. P. Seitsonen, and F. Mauri, Phys. Rev. B 80, 075102 (2009).

[46] O. Bunau and M. Calandra, Phys. Rev. B 87, 205105 (2013).

[47] E. Diesen, G. L. S. Rodrigues, A. C. Luntz, F. Abild-Pedersen, L. G. M. Pettersson, and J. Voss, AIP Adv. 10, 115014 (2020).

[48] R. J. Maurer, M. Askerka, V. S. Batista, and J. C. Tully, Phys. Rev. B 94, 115432 (2016).

[49] A. Föhlisch, P. Feulner, F. Hennies, A. Fink, D. Menzel, D. Sanchez-Portal, P. M. Echenique, and W. Wurth, Nature (London) 436, 373 (2005).

[50] H. Öberg, J. Gladh, K. Marks, H. Ogasawara, A. Nilsson, L. G. M. Pettersson, and H. Öström, J. Chem. Phys. 143, 074701 (2015).

[51] M. Lisowski, P. A. Loukakos, U. Bovensiepen, J. Stähler, C. Gahl, and M. Wolf, Appl. Phys. A 78, 165 (2004).

[52] C. Benndorf, E. Bertel, V. Dose, W. Jacob, N. Memmel, and J. Rogozik, Surf. Sci. 191, 455 (1987).

[53] J. A. Misewich, T. F. Heinz, and D. M. Newns, Phys. Rev. Lett. 68, 3737 (1992).

[54] D. Novko, M. Alducin, and J. I. Juaristi, Phys. Rev. Lett. 120, 156804 (2018).

[55] D. Novko, J. C. Tremblay, M. Alducin, and J. I. Juaristi, Phys. Rev. Lett. 122, 016806 (2019). 